\title{
How to move a black hole without excision: gauge conditions for the numerical evolution of a moving puncture
}

\author{
James R. van Meter, ${ }^{1}$ John G. Baker ${ }^{1}$ Michael Koppitz, ${ }^{1}$ and Dae-Il Choi ${ }^{1,2}$ \\ ${ }^{1}$ Gravitational Astrophysics Laboratory, NASA Goddard Space Flight Center, 8800 Greenbelt Rd., Greenbelt, MD 207r1, USA \\ ${ }^{2}$ Universities Space Research Association, 10211 Wincopin Circle, Suite 500, Columbia, MD 21044
}

\begin{abstract}
Recent demonstrations of unexcised black holes traversing across computational grids represent a significant advance in numerical relativity. Stable and accurate simulations of multiple orbits, and their radiated waves, result. This capability is critically undergirded by a careful choice of gauge. Here we present analytic considerations which suggest certain gauge choices, and numerically demonstrate their efficacy in evolving a single moving puncture black hole.
\end{abstract}

PACS numbers:

\section{INTRODUCTION}

Gravitational waveforms from binary black hole mergers can only be obtained through 3D numerical relativity simulations of the full Einstein equations. Such simulations have proven to be very challenging. Improvements have often come from new formulations or a new choice of gauge. For example, a corotating gauge led to the first evolution of a binary through one full orbit [1] as well as through a plunge, merger, and ringdown [2].

Recently, the authors of this paper [3] and another research group [4] independently developed the capability to numerically evolve freely moving black holes, i.e. black holes that are in no way fixed to any position in the computational grid, without using excision techniques for the black hole interiors. These efforts led to the simulation of record-breaking numbers of orbits, among other accomplishments [5]. Both of these research programs employed gauge conditions that differed not only from those prescribed in prior literature, but also from each other. A careful choice of gauge is important, particularly for moving black holes, as it can determine whether a simulation is stable or becomes irrevocably corrupted by a runaway build up of numerical error. There are various ways in which a choice of gauge can affect the stability and accuracy of a numerical simulation; for example, a poor choice of gauge might cause large gradients in the fields which in turn engender large finite differencing error.

In this paper we investigate a group of gauge choices analytically, and numerically demonstrate their effects on moving black hole simulations. These gauge choices are framed in the context of a conventional, $3+1$, conformal formulation of Einstein's equations known as the Baumgarte-Shapiro-Shibata-Nakamura (BSSN) formulation $[6,6,[8,9]$. After a brief exploration of the shortcomings of a commonly used gauge choice in III we calculate in section $\amalg A$ the characteristic speeds for various gauges and then show the numerical behavior of some of them in section [IIB In section [V] we discuss the properties of the most promising gauge choices and suggest further improvements. Conclusions are presented in section $\nabla$

\section{ORIGINAL 1+LOG SLICING AND GAMMA-DRIVER SHIFT CONDITIONS}

We consider first a gauge introduced in [10] for evolving non-moving punctures without excision:

$$
\begin{aligned}
\partial_{t} \alpha & =-2 \alpha K \\
\partial_{t} \beta^{i} & =\frac{3}{4} \alpha \Psi_{0}^{-n} B^{i} \\
\partial_{t} B^{i} & =\partial_{t} \tilde{\Gamma}^{i}-\eta B^{i}
\end{aligned}
$$

where $\alpha$ is the lapse, $\beta^{i}$ is the shift, $K$ and $\tilde{\Gamma}^{i}$ are the usual BSSN variables, $\Psi_{0}^{4}$ is the initial conformal factor $\left(\Psi_{B L}^{4}\right.$ in [10] $), B^{i}$ is an auxiliary variable to make the shift equation first order in time, and $\eta$ is a constant damping factor. This gauge has proven to be very effective at evolving a single non-moving puncture for arbitrarily long durations, and in some circumstances it can also be applied to binary punctures. Here the condition on the lapse, a variation of the Bona-Masso slicing condition 10, 11], is the standard " $1+\log$ " singularity-avoiding slicing condition. Of particular interest is the "Gammadriver" condition for the shift, which is designed to ultimately "freeze Gamma", i.e. drive $\partial_{t} \tilde{\Gamma}$ to zero as the physics of the spacetime also evolve towards quiescence.

For $n>0, \Psi_{0}^{-n}=0$ at the puncture, and thus this specific gauge will ensure that $\beta^{i}$ will not evolve there. As the motion of the puncture is determined by the shift, the puncture will remain motionless in this gauge since the shift is initially zero. For black holes that are physically dynamical the coordinates may become highly distorted. For example, in the case of binary black holes, the separation between the black holes as determined by the proper distance from horizon to horizon in some spatial slice must decrease with time. This implies that components of the 3-metric will approach zero between the black holes. Then the evolved part of the BSSN conformal exponent $\phi$ must either approach $-\infty$, or components of the conformal 3-metric $\tilde{\gamma}_{i j}$ must approach zero. In the latter case the inverse 3 -metric must diverge in order to maintain a unit determinant. Thus, in the fixed puncture approach, arbitrarily large, ever-increasing fields and gradients around the black holes seem likely to develop and 
cause numerical difficulties. Indeed this can be demonstrated even in the case of a single moving black hole.

We will use as a test case a single black hole, given by Bowen-York puncture initial data 12] (as computed by the elliptic solver AMRMG [13]), with unit puncture mass and momentum such that its physical speed should be half the speed of light. We evolve this data with our Hahndol code [9], which uses the usual, conformal BSSN formulation of Einstein's evolution equations on a cellcentered numerical grid, with $4^{\text {th }}$-order spatial differencing and $4^{\text {th }}$-order Runge-Kutta time-integration. The initial puncture position is at coordinates $(-3 M, 0,0)$ (where $M$ is the puncture mass) and the momentum is in the positive $x$-direction. The $x$-axis is between grid points, which are a distanced $M / 16$ apart; all data presented here has been interpolated onto the $x$-axis for plotting purposes. $\beta^{i}$ and $B^{i}$ are initialized to zero, while the lapse is precollapsed with the profile $\Psi_{0}^{-2}$ as suggested in [10] (and recommended in [4] for moving punctures). The damping parameter $\eta$ is 2 , unless otherwise stated. We will assess the performance of each gauge by the evolving behavior of the quantities $\alpha, \beta^{x}, \phi$, and $\tilde{\Gamma}^{x}$; in particular the peak in $\phi$ will roughly indicate the position of the puncture and any extreme gradients in $\tilde{\Gamma}^{x}=-\partial_{j} \tilde{\gamma}^{j x}$ will tend to indicate problems with the gauge.

In this regard it should be noted that $\tilde{\Gamma}^{i}$ is completely controllable by the gauge condition, and so any undesirable features observed in $\tilde{\Gamma}^{i}$ are in principle avoidable via a better choice of gauge. In particular, the specific "Gamma-freezing" condition $\tilde{\Gamma}^{i}=0$ may be desirable, as this elliptic generalization of isotropic coordinates proposed by Dirac is expected to be non-pathological [14, 15]. So, among hyperbolic Gamma-driving conditions, those that result in smaller values of $\tilde{\Gamma}^{i}$ and its derivatives might be preferred.

In Fig. 11 evolving with the original gauge given in Eqs. (13), we see that $\tilde{\Gamma}^{x}$ grows very large and $\phi$ develops sharp features. Shortly thereafter the inverse conformal metric diverges, which is our criterion for stopping the run. This failure motivates investigation of moving punctures.

If we wish the punctures to move we must allow $\beta^{i}$ to evolve freely away from zero and thus we must take the conformal factor out of the shift equation (setting $n=0$ in Eq. (2)). The results, shown in Fig. 2] are not much improved. The puncture is now free to move but non-propagating features at $x=-3 M$ destabilize the simulation. Clearly there is a problem with zero-speed modes. The extended, collapsed region of the lapse points to a particular difficulty in this regard, and suggests an improvement.

The factor of $\alpha$ in Eq. (2) should also be removed, for even if it were not precollapsed it would still be driven nearly to zero around the puncture by the $1+\log$ slicing condition, and therefore may retard the evolution of $\beta^{i}$. When this factor is removed, the results are not as catastrophic as before, but the simulation is plagued with noise. Fig. [3]shows, in particular, a tendency for the min-

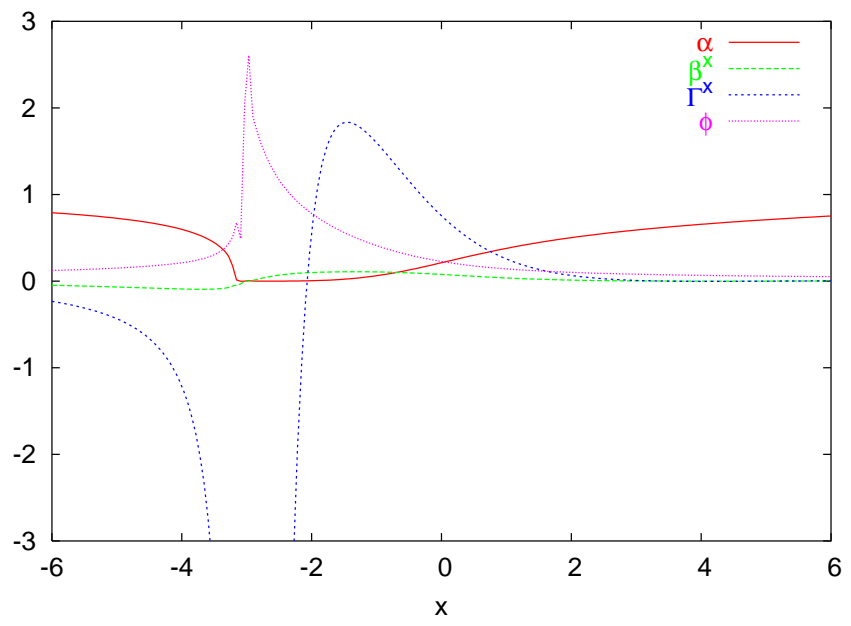

FIG. 1: Results of the non-moving puncture gauge $\partial_{t} \alpha=$ $-2 \alpha K, \partial_{t} \beta^{i}=\frac{3}{4} \alpha \Psi_{0}^{-n} B^{i}, \partial_{t} B^{i}=\dot{\tilde{\Gamma}}^{i}-\eta B^{i}$ at time $t=17 M$. The conformal metric becomes singular by $t=18 \mathrm{M}$.

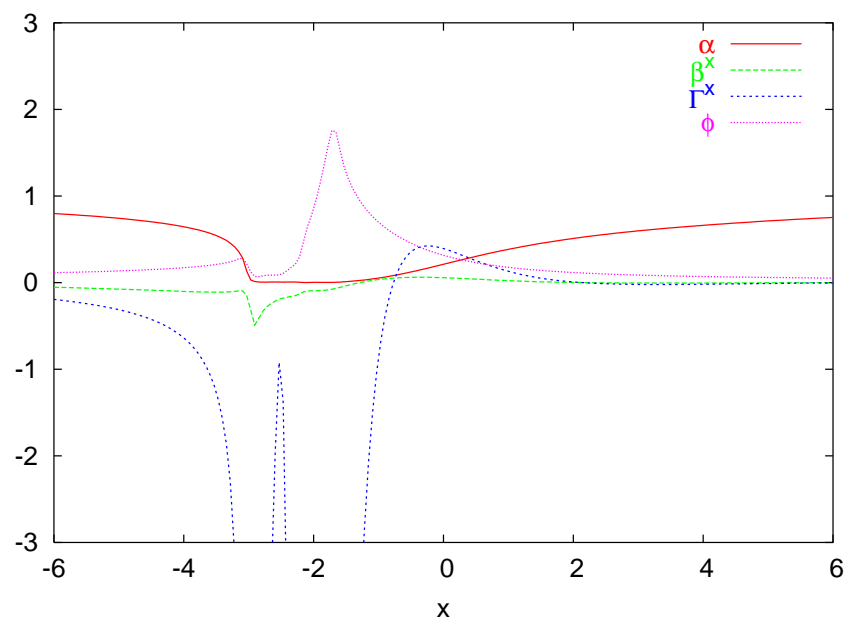

FIG. 2: Results of the gauge $\partial_{t} \alpha=-2 \alpha K, \partial_{t} \beta^{i}=\frac{3}{4} \alpha B^{i}$, $\partial_{t} B^{i}=\dot{\tilde{\Gamma}}^{i}-\eta B^{i}$ at time $t=17 M$. Sharp features around $x=-3 M$ fail to propagate. The conformal metric becomes singular by $t=18 M$.

imum in the lapse to lag behind the puncture position. The above results suggest that a careful study of the propagation speeds in this system might be helpful.

\section{CHARACTERISTIC SPEEDS}

\section{A. Eigenvalue analysis}

A potential danger of zero-speed modes is that errors may couple to them, compound in place, and become inordinantly large. Zero-speed modes seem particularly hazardous in the case of moving black holes as they may adversely affect the dynamics by putting a drag on the 

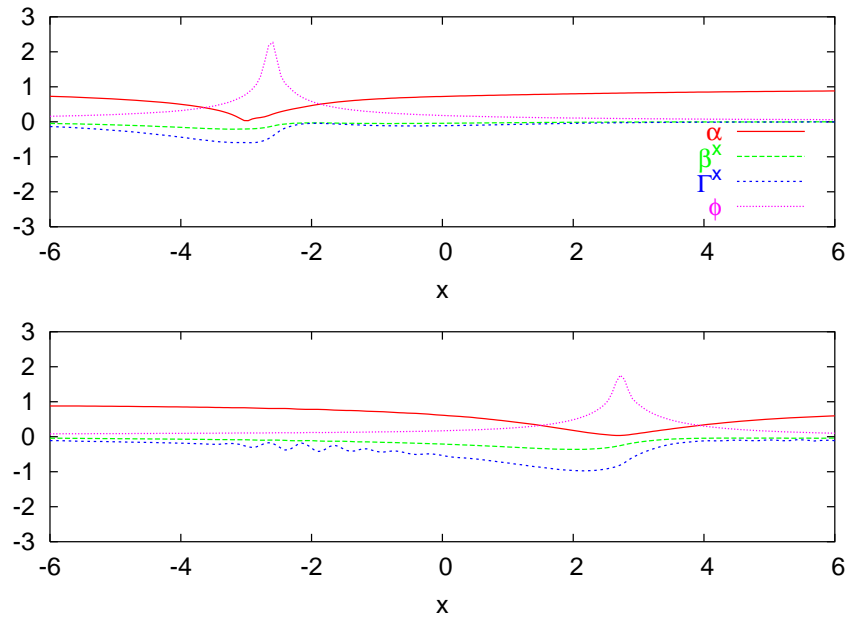

FIG. 3: Results of the gauge $\partial_{t} \alpha=-2 \alpha K, \partial_{t} \beta^{i}=\frac{3}{4} B^{i}$, $\partial_{t} B^{i}=\dot{\tilde{\Gamma}}^{i}-\eta B^{i}$ at times $t=4.5 M$ (top panel) and $t=30 M$ (bottom panel). The top panel clearly shows the lapse lagging behind the puncture, while the bottom panel exhibits noise propagating from the puncture.

puncture motion. In some cases zero-speed modes can also be a source of instability [16]. So there is good reason to be aware of such modes.

For the purpose of investigating characteristic speeds, a simple yet revealing method of analysis is to linearize the equations, assume plane wave solutions, and solve for the eigenmodes of the resulting algebraic system. With the gauge conditions described above, the BSSN equations can be linearized about flat space as follows.

$$
\begin{aligned}
\dot{a}= & -2 \alpha_{0} K \\
\dot{B}^{i}= & \dot{\tilde{\Gamma}}^{i} \\
\dot{b}^{i}= & \frac{3}{4} \alpha_{0}^{p} B \\
\dot{\phi}= & -\frac{1}{6}\left(\alpha_{0} K-\partial_{i} b^{i}\right)+\beta_{0}^{k} \partial_{k} \phi \\
\dot{K}= & -\partial_{i} \partial_{i} a+\beta_{0}^{k} \partial_{k} K \\
\dot{h}_{i j}= & -2 \alpha_{0} \tilde{A}_{i j}+\partial_{i} b^{j}+\partial_{j} b^{i}-\frac{2}{3} \delta_{i j} \partial_{k} b^{k}+\beta_{0}^{k} \partial_{k} h_{i j}(9) \\
\dot{\tilde{A}}_{i j}= & {\left[-\partial_{i} \partial_{j} a-\frac{1}{2} \alpha_{0} \partial_{k} \partial_{k} h_{i j}+\frac{1}{2} \alpha_{0} \partial_{i} \tilde{\Gamma}^{j}+\frac{1}{2} \alpha_{0} \partial_{j} \tilde{\Gamma}^{i}\right.} \\
& \left.-2 \alpha_{0} \partial_{i} \partial_{j} \phi\right]^{\mathrm{TF}}+\beta_{0}^{k} \partial_{k} \tilde{A}_{i j} \\
\dot{\tilde{\Gamma}}^{i}= & -\frac{4}{3} \alpha_{0} \partial_{i} K+\partial_{k} \partial_{k} b^{i}+\frac{1}{3} \partial_{i} \partial_{j} b^{j}+\beta_{0}^{k} \partial_{k} \tilde{\Gamma}^{i}
\end{aligned}
$$

where $a \equiv \alpha-\alpha_{0}, b^{i} \equiv \beta^{i}-\beta_{0}^{i}, h_{i j} \equiv \tilde{\gamma}_{i j}-\delta_{i j}$, and the damping term on $B^{i}$ has been dropped as it should not affect the real part of the characteristic speeds. The lapse and shift are assumed to have constant, zeroth order terms $\alpha_{0}$ and $\beta_{0}^{i}$, respectively which conveniently allows us to capture essential effects of the lapse factors and the advection terms without making the eigenvalue problem intractable.

For spatial variation only in one dimension, with no initial transverse components, and assuming plane-wave solutions, the above system of equations can be written in the form:

$$
\partial_{t}|u\rangle=i k \mathrm{M}|u\rangle
$$

where

$$
|u\rangle=\left(\begin{array}{c}
\hat{a} \\
\hat{B} \\
\hat{b} \\
\hat{\phi} \\
\hat{K} \\
\hat{h} \\
\hat{A} \\
\hat{\Gamma}
\end{array}\right) e^{i(k x-\omega t)}
$$

with $\hat{a}, \hat{B}, \hat{b}, \hat{\phi}, \hat{K}, \hat{h}, \hat{A}$, and $\hat{\Gamma}$ the constant amplitudes of $a, B^{x}, b^{x}, \phi, K, h_{x x}, \tilde{A}_{x x}$, and $\tilde{\Gamma}^{x}$ respectively, and $\mathrm{M}=$

$$
\left(\begin{array}{cccccccc}
0 & 0 & 0 & 0 & -\frac{2}{i k} \alpha_{0} & 0 & 0 & 0 \\
0 & 0 & \frac{4 i k}{3} & 0 & \frac{4}{3} \alpha_{0} & 0 & 0 & -\beta_{0} \\
0 & \frac{3 \alpha_{0}^{p}}{4 i k} & 0 & 0 & 0 & 0 & 0 & 0 \\
0 & 0 & -\frac{1}{6} & -\beta_{0} & -\frac{1}{6 i k} \alpha_{0} & 0 & 0 & 0 \\
-i k & 0 & 0 & 0 & -\beta_{0} & 0 & 0 & 0 \\
0 & 0 & -\frac{4}{3} & 0 & 0 & -\beta_{0} & -\frac{2}{i k} \alpha_{0} & 0 \\
-\frac{2 i k}{3} & 0 & 0 & -\frac{4 i k}{3} \alpha_{0} & 0 & -\frac{i k}{2} \alpha_{0} & -\beta_{0} & -\frac{2}{3} \alpha_{0} \\
0 & 0 & \frac{4 i k}{3} & 0 & \frac{4}{3} \alpha_{0} & 0 & 0 & -\beta_{0}
\end{array}\right)
$$

The eigenvalues of $\mathrm{M}$ are $0,-\beta_{0},-\beta_{0}+\alpha_{0},-\beta_{0}-$ $\alpha_{0},-\frac{1}{2} \beta_{0}+\frac{1}{2} \sqrt{\beta_{0}^{2}+8 \alpha_{0}},-\frac{1}{2} \beta_{0}-\frac{1}{2} \sqrt{\beta_{0}^{2}+8 \alpha_{0}},-\frac{1}{2} \beta_{0}+$ $\frac{1}{2} \sqrt{\beta_{0}^{2}+4 \alpha_{0}^{p}}$, and $-\frac{1}{2} \beta_{0}-\frac{1}{2} \sqrt{\beta_{0}^{2}+4 \alpha_{0}^{p}}$. If $|v\rangle$ is the eigenvector associated with eigenvalue $v$, and the covector $\langle v|$ is defined such that $\sum_{v}|v\rangle\langle v|=\mathrm{I}$, then the eigen decomposition of $\mathrm{M}$ allows the system of evolution equations to be written as a series of advection terms, each associated with a characteristic velocity equal to one of the eigenvalues:

$$
\partial_{t}|u\rangle=\sum_{v} v \partial_{x}|v\rangle\langle v \mid u\rangle
$$

The inner product with $\langle v|$ gives:

$$
\partial_{t}\langle v \mid u\rangle=v \partial_{x}\langle v \mid u\rangle
$$

Now we are interested in slow speed modes. Note that although $\beta^{i}$ is typically initialized at zero, with the Gamma-driver condition it evolves rapidly and significantly enough from zero that we do not classify $\beta_{0}$ as a slow mode (since we're interested in moving punctures, $\beta^{i}$ is generally non-vanishing). In addition to the obvious zero speed mode there are two modes that approach zero in the limit where $\alpha_{0}$ approaches zero. If $\left|\alpha_{0}\right|<<\left|\beta_{0}\right|$ then $-\frac{1}{2} \beta_{0}+\frac{1}{2} \sqrt{\beta_{0}^{2}+8 \alpha_{0}}$ and $-\frac{1}{2} \beta_{0}+\frac{1}{2} \sqrt{\beta_{0}^{2}+4 \alpha_{0}^{p}}$ become approximately $4 \alpha_{0} / \beta_{0}$ and $2 \alpha_{0}^{p} / \beta_{0}$, respectively. In 
the full nonlinear system, the $1+\log$ slicing condition will collapse $\alpha$ nearly to zero in a finite region around the puncture (where $\beta^{i}$ will be nonzero for a moving puncture). Modes which propagate at a speed approximately proportional to a positive power of $\alpha$ for small $\alpha$, which we will refer to as $\alpha$-speed modes, are thus of potential concern. In particular, late in the evolution of black hole binaries there will emerge a significant, stationary region in which the lapse has collapsed nearly to zero, in which $\alpha$-speed modes are effectively zero-speed modes.

The eigencovector for $v=-\frac{1}{2} \beta_{0}+\frac{1}{2} \sqrt{\beta_{0}^{2}+8 \alpha_{0}}$ involves only the fields $a$ and $K$, is relatively independent from the other fields, and easy to fix, so we will address it first. This $\alpha$-speed mode can be understood also in the context of the original nonlinear equations by noting that $\alpha$ couples with $K$ to give a wave equation such that the speed of fluctuations of the lapse goes to zero when the lapse itself goes to zero. It is particularly egregious when the lapse couples to an $\alpha$-speed mode, as once it collapses nearly to zero it will tend to get "stuck" there. But this can be remedied in a natural way simply by adding an advection term as follows,

$$
\dot{\alpha}=-2 \alpha K+\beta^{j} \partial_{j} \alpha
$$

which will modify the principal part of its wave equation such that when $\alpha$ goes to zero its speed will go to $\beta$. Note that Eq. (17), first used for moving punctures in [4], is consistent with the original Bona-Mass family of slicing conditions 11].

The eigencovector for $-\frac{1}{2} \beta_{0}+\frac{1}{2} \sqrt{\beta_{0}^{2}+4 \alpha_{0}^{p}}$ is more complicated, involving not only $a$ but several other fields as well. It turns out that advecting the lapse will not remove this mode. But noting that this eigenfield involves $b^{x}$, and that the equation for $b^{x}$ is conspicuously absent an advection term, an obvious stratagem is to advect the original $\beta^{i}$ as follows:

$$
\dot{\beta}^{i}=\frac{3}{4} B^{i}+\beta^{j} \partial_{j} \beta^{i}
$$

Finally, Eq. (16) for the eigenvalue $v=0$ yields

$$
\partial_{t}\left(B^{x}-\tilde{\Gamma}^{x}\right)=0
$$

This equation, a trivial consequence of the original Gamma-driver condition, immediately suggests a simple modification. The most natural way to remove this zerospeed mode is to advect it at $\beta$ speed by modifying the $\dot{B}^{i}$ equation thus:

$$
\partial_{t} B^{i}=\partial_{t} \tilde{\Gamma}^{i}+\beta^{j} \partial_{j}\left(B^{i}-\tilde{\Gamma}^{i}\right)-\eta B^{i}
$$

It turns out that the $+\beta^{j} \partial_{j} B^{i}$ term alone will not suffice, as for example it might introduce an exponentially growing mode. Meanwhile the $-\beta^{j} \partial_{j} \tilde{\Gamma}^{i}$ term alone adds another $\alpha$-speed mode to the system.

The foregoing analysis suggests the addition of four advection terms to the gauge equations, as indicated in
Eqs. (171820). The lapse equation is relatively independent of the others: if it is advected then a particular $\alpha$-speed mode is removed, and if it is not advected then this particular $\alpha$-speed mode remains. But the three advection terms suggested for the shift equations are strongly interdependent, so their combined effects are not so obvious and it is instructive to consider which undesirable modes result from which combinations of these three terms. The possible combinations and their resulting "bad" speeds are summarized in Table 1. Here we see that for $p>0$, only when all the above advection terms are included will the system be free of slowspeed and exponentially growing modes; while for $p=0$, the $-\beta^{j} \partial_{j} \tilde{\Gamma}^{i}$ term remains critical. In the case with all advection terms the benign eigenspeeds are: $-\beta_{0},-\beta_{0}$, $-\beta_{0}-\alpha_{0},-\beta_{0}+\alpha_{0},-\beta_{0}-\sqrt{\alpha_{0}^{p}},-\beta_{0}+\sqrt{\alpha_{0}^{p}},-\beta_{0}-\sqrt{2 \alpha_{0}}$, $-\beta_{0}+\sqrt{2 \alpha_{0}}$ (where $-\beta_{0}$ is listed twice because it is now associated with two distinct eigenvectors).

\begin{tabular}{|c|c|c|c|l|}
\hline Case \# & $+\beta^{j} \partial_{j} B^{i}$ & $-\beta^{j} \partial_{j} \tilde{\Gamma}^{i}$ & $+\beta^{j} \partial_{j} \beta^{i}$ & "bad" speeds \\
\hline \hline 1 & $\mathrm{~N}$ & $\mathrm{~N}$ & $\mathrm{~N}$ & $0, \alpha^{p}$ \\
\hline 2 & $\mathrm{~N}$ & $\mathrm{~N}$ & $\mathrm{Y}$ & 0 \\
\hline 3 & $\mathrm{~N}$ & $\mathrm{Y}$ & $\mathrm{N}$ & $\alpha^{p / 2}$ \\
\hline 4 & $\mathrm{~N}$ & $\mathrm{Y}$ & $\mathrm{Y}$ & $\alpha^{p}$ \\
\hline 5 & $\mathrm{Y}$ & $\mathrm{N}$ & $\mathrm{N}$ & 0 \\
\hline 6 & $\mathrm{Y}$ & $\mathrm{N}$ & $\mathrm{Y}$ & $-i$ \\
\hline 7 & $\mathrm{Y}$ & $\mathrm{Y}$ & $\mathrm{N}$ & $\alpha^{p}$ \\
\hline 8 & $\mathrm{Y}$ & $\mathrm{Y}$ & $\mathrm{Y}$ & none \\
\hline
\end{tabular}

TABLE I: Effect of various advection terms in the shift equations on the presence of undesirable eigenspeeds. ' $\mathrm{Y}$ ' or ' $\mathrm{N}$ ' indicates whether each advection term is included or not, respectively, in Eq. (18) or Eq. (20). 0, $\alpha^{p}$, or $-i$ indicates whether the resulting linearized equations have a zero-speed mode, an $\alpha$-speed mode, or an exponentially growing mode, respectively. The lapse is assumed to be advected as in Eq. (17); otherwise an additional $\alpha$-speed mode would appear in every case.

\section{B. Numerical tests}

Evidence of the slow-speed modes found in the linearized analysis can often (but not always) be found in simulations of the full nonlinear system. Note that zerospeed modes only represent a potential danger. In some cases errors may not couple to these modes even though they exist in the equations.

Here we provide a few examples of the cases given in Table I Fig. 4 represents Case \#1 with $p=1$, which is predicted to have a zero-speed mode and indeed manifests a non-propagating feature at $x=-3 M$. On the other hand, Case \#1 with $p=0$, also predicted to have a zero-speed mode, belies no indication of it in Fig. 5 


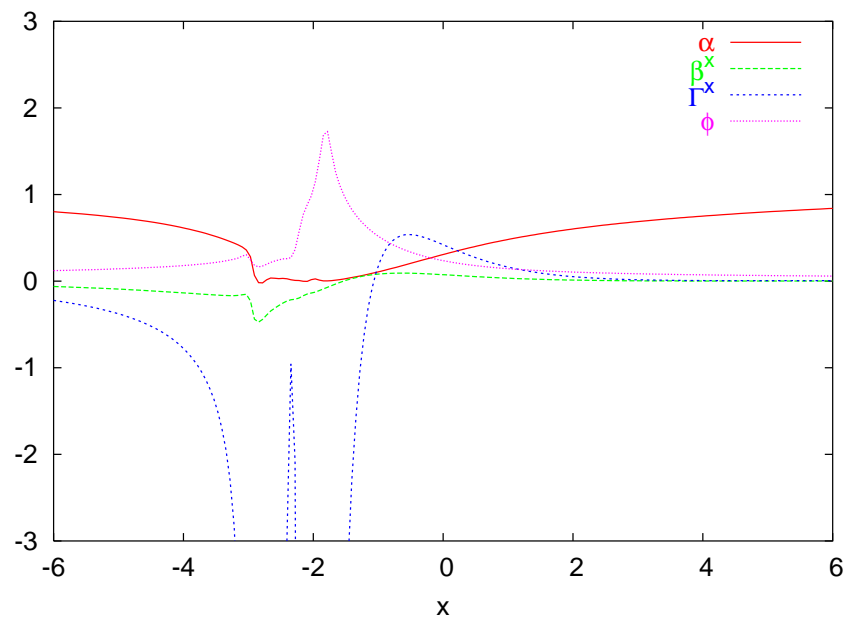

FIG. 4: Results for the gauge $\partial_{t} \alpha=-2 \alpha K+\beta^{j} \partial_{j} \alpha, \partial_{t} \beta^{i}=$ $\frac{3}{4} \alpha B^{i}, \partial_{t} B^{i}=\partial_{t} \tilde{\Gamma}^{i}-\eta B^{i}$ (Case \#1 in Table 1 with $\mathrm{p}=1$ ) at time $t=14 M$. Non-propagating features are evident around $x=-3 M$. The conformal metric becomes singular by $t=$ $15 M$

instead appearing quite smooth. In this case, evidently, the zero-speed mode does not couple significantly with the other fields. In Fig. [6] the spike in $\tilde{\Gamma}^{x}$, which becomes steeper in time as the "tail" in $\tilde{\Gamma}^{x}$ grows, is apparently unrelated to slow-speed modes as none are predicted in this Case \#3 with $p=0$. However, for a longer evolution this growing gradient in $\tilde{\Gamma}^{x}$ can be expected to adversely affect the constraints. In Fig. [7 the tail in $\tilde{\Gamma}^{x}$ and in particular the non-propagating bend in the tail around $x=-2 M$ seems to be evidence of the zero-speed mode predicted for Case \#5. In Fig. 8 noise in $\tilde{\Gamma}^{x}$ grows exponentially, as expected for Case \#6. And finally, Case \#8, depicted in Fig. 9] and Fig. 10, is demonstrated to be free of the previously identified "bad" speed modes whether $p=0$ or $p=1$. (Although the former appears much smoother, perhaps because the eigenspeeds $-\beta_{0} \pm 1$ allow faster propagation of error away from the puncture than $-\beta_{0} \pm \sqrt{\alpha_{0}}$.)

\section{PROPERTIES OF THE "CLEANEST" GAUGES}

Of all the combinations suggested in Table two distinguish themselves in numerical tests as conducive to particularly smooth propagation of the moving black hole. These gauges are Case \#8 with $p=0$, which we will refer to as the "shifting-shift case":

$$
\begin{aligned}
\partial_{t} \beta^{i}-\beta^{j} \partial_{j} \beta^{i} & =\frac{3}{4} B^{i} \\
\partial_{t} B^{i}-\beta^{j} \partial_{j} B^{i} & =\partial_{t} \tilde{\Gamma}^{i}-\beta^{j} \partial_{j} \tilde{\Gamma}^{i}-\eta B^{i}
\end{aligned}
$$

and Case \#1 with $p=0$, which we will refer to as the "non-shifting-shift" case:

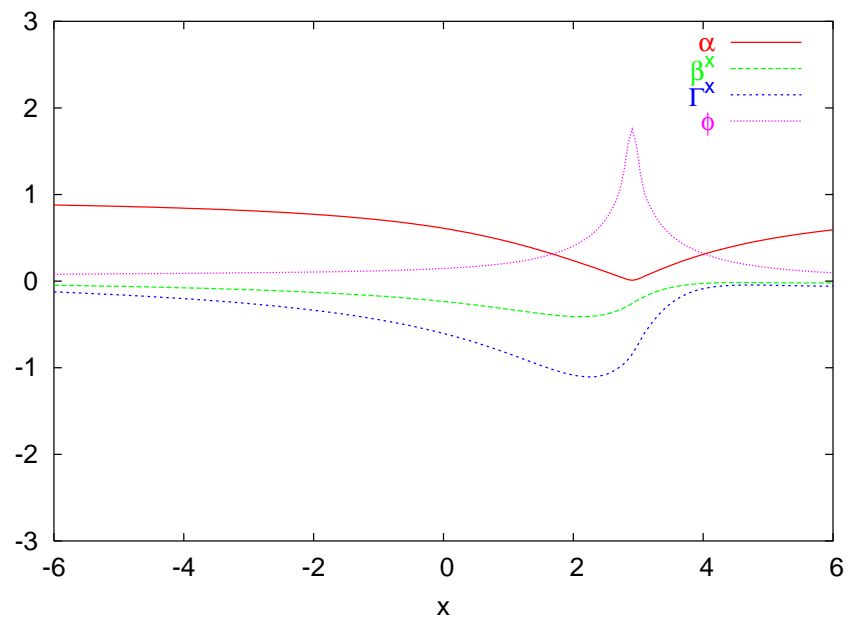

FIG. 5: Results for the gauge $\partial_{t} \alpha=-2 \alpha K+\beta^{j} \partial_{j} \alpha, \partial_{t} \beta^{i}=$ $\frac{3}{4} B^{i}, \partial_{t} B^{i}=\partial_{t} \tilde{\Gamma}^{i}-\eta B^{i}$ (Case \#1 in Table \with $\mathrm{p}=0$ ) at time $t=30 \mathrm{M}$. The evolution appears smooth.

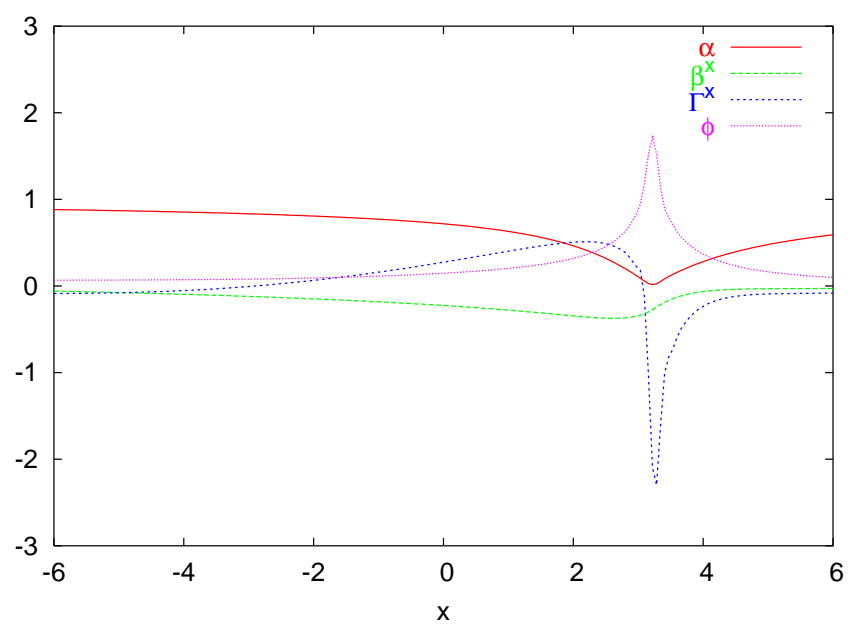

FIG. 6: Results for the gauge $\partial_{t} \alpha=-2 \alpha K+\beta^{j} \partial_{j} \alpha, \partial_{t} \beta^{i}=$ $\frac{3}{4} \alpha B^{i}, \partial_{t} B^{i}=\partial_{t} \tilde{\Gamma}^{i}-\beta^{j} \partial_{j} \tilde{\Gamma}^{i}-\eta B^{i}$ (Case \#3 in Table \with $\mathrm{p}=0)$ at time $t=30 M$. $\tilde{\Gamma}^{x}$ continues to grow larger and its gradient steeper.

$$
\begin{aligned}
\partial_{t} \beta^{i} & =\frac{3}{4} B^{i} \\
\partial_{t} B^{i} & =\partial_{t} \tilde{\Gamma}^{i}-\eta B^{i}
\end{aligned}
$$

The efficacy of Eqs. (23 24) in evolving moving punctures was originally demonstrated in [4, 17]. Apparently the zero-speed mode is not problematical in this case. Regarding the "shifting-shift" condition, Eqs. (21 22), we have recently used it successfully in the stable evolution of two equal mass black holes through 5.5 orbits. This latter gauge has also been recommended for the strong hyperbolicity it brings to the BSSN equations [18, 19], suggesting it may be a more robustly stable choice than Eqs. (23 24). Nevertheless for the single black hole sim- 


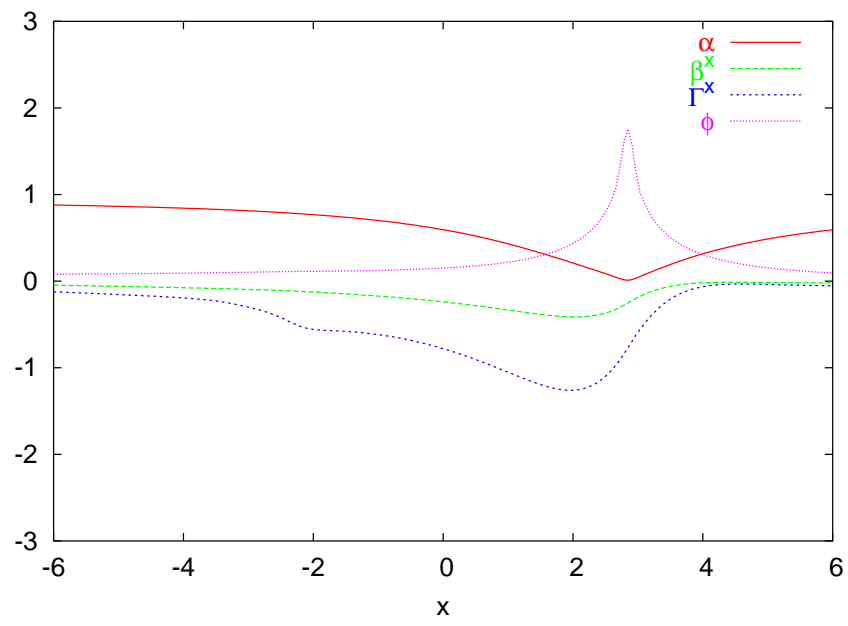

FIG. 7: Results for the gauge $\partial_{t} \alpha=-2 \alpha K+\beta^{j} \partial_{j} \alpha, \partial_{t} \beta^{i}=$ $\frac{3}{4} \alpha B^{i}, \partial_{t} B^{i}=\partial_{t} \tilde{\Gamma}^{i}+\beta^{j} \partial_{j} B^{i}-\eta B^{i}$ (Case \#5 in Table 【with $\mathrm{p}=0)$ at time $t=30 M$. The feature around $x=-2 M$ does not propagate.

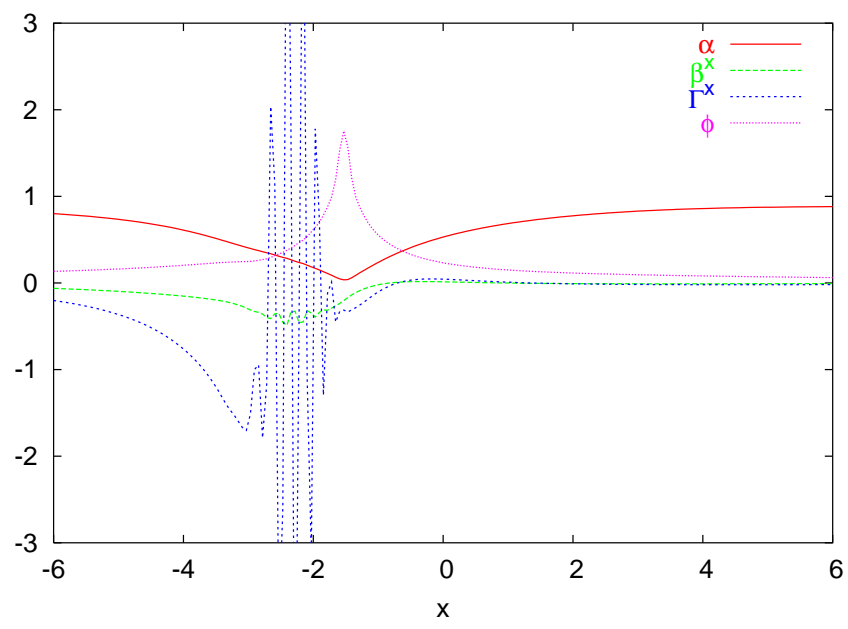

FIG. 8: Results for the gauge $\partial_{t} \alpha=-2 \alpha K+\beta^{j} \partial_{j} \alpha, \partial_{t} \beta^{i}=$ $\frac{3}{4} \alpha B^{i}+\beta^{j} \partial_{j} \beta^{i}, \partial_{t} B^{i}=\partial_{t} \tilde{\Gamma}^{i}+\beta^{j} \partial_{j} B^{i}-\eta B^{i}$ (Case \#6 in Table \with $\mathrm{p}=0$ ) at time $t=10 M$ The noise in $\tilde{\Gamma}^{x}$ grows exponentially. The conformal metric becomes singular by $t=$ $11 M$.

ulations represented by Figs. [5 and [10, the results from these two gauge choices are very similar.

For these two gauge choices, we now consider varying the damping parameter, which has up to now been set to $\eta=2$. By moving toward $\eta \rightarrow 0$ Eqs. 2122) appear a little closer to realizing a "Gamma-freezing" condition. Indeed, with $\eta=0$, we find that the the black holes come closer to realizing the physically expected velocity. Both the "shifting-shift" and "non-shifting-shift" gauge options with $\eta=0$ have proven to allow stable evolutions of a single black hole. In Fig.[1] we find that in both cases setting $\eta=0$ results in significantly smaller values for $\tilde{\Gamma}^{i}$, meaning a closer approximation to the Dirac gauge.

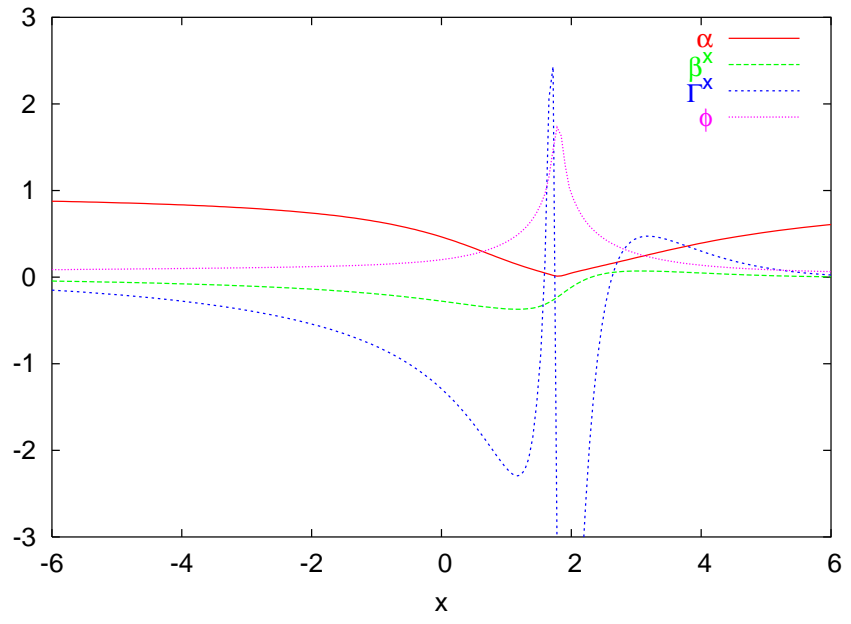

FIG. 9: Results for the gauge $\partial_{t} \alpha=-2 \alpha K+\beta^{j} \partial_{j} \alpha, \partial_{t} \beta^{i}=$ $\frac{3}{4} \alpha B^{i}+\beta^{j} \partial_{j} \beta^{i}, \partial_{t} B^{i}=\partial_{t} \tilde{\Gamma}^{i}+\beta^{j} \partial_{j}\left(B^{i}-\tilde{\Gamma}^{i}\right)-\eta B^{i}$ (Case \#8 in Table【with $\mathrm{p}=1$ ) at time $t=30 M$. Aside from the sharp features in $\tilde{\Gamma}^{i}$, which do not grow significantly in time, the propagation is non-pathological.

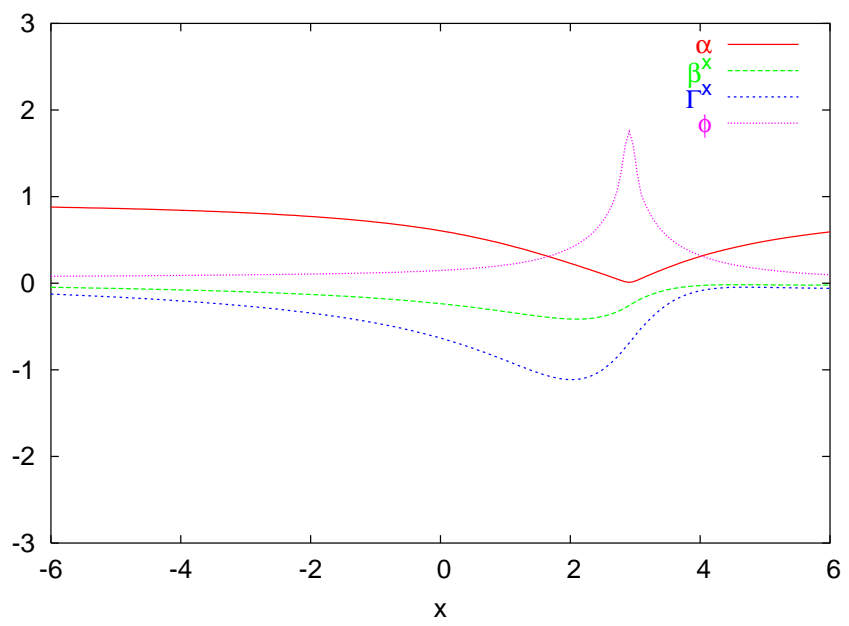

FIG. 10: Results for the gauge $\partial_{t} \alpha=-2 \alpha K+\beta^{j} \partial_{j} \alpha, \partial_{t} \beta^{i}=$ $\frac{3}{4} B^{i}+\beta^{j} \partial_{j} \beta^{i}, \partial_{t} B^{i}=\partial_{t} \tilde{\Gamma}^{i}+\beta^{j} \partial_{j}\left(B^{i}-\tilde{\Gamma}^{i}\right)-\eta B^{i}$ (Case \#8 in Table $\$ with $\mathrm{p}=0$ ) at time $t=30 M$. The evolution is very smooth.

Further, we comment that we have observed numerically that for $\eta>0, \tilde{\Gamma}^{i}$ appears to show very slow linear growth in time, whereas for $\eta=0, \tilde{\Gamma}^{i}$ appears to be bounded. Comparing the "shifting-shift" and "non-shifting-shift" options with $\eta=0$ we now find more noticeable differences in $\tilde{\Gamma}^{i}$, with our recommended "shifting-shift" option giving a smoother result with $\tilde{\Gamma}^{i}$ holding closer to zero near the puncture.

These two gauges share an additional feature in common, which is that either Eqs. (2324) or Eqs. (2122) can be integrated to give the relation

$$
B^{i}=\tilde{\Gamma}^{i}-\frac{4}{3} \eta \beta^{i}
$$




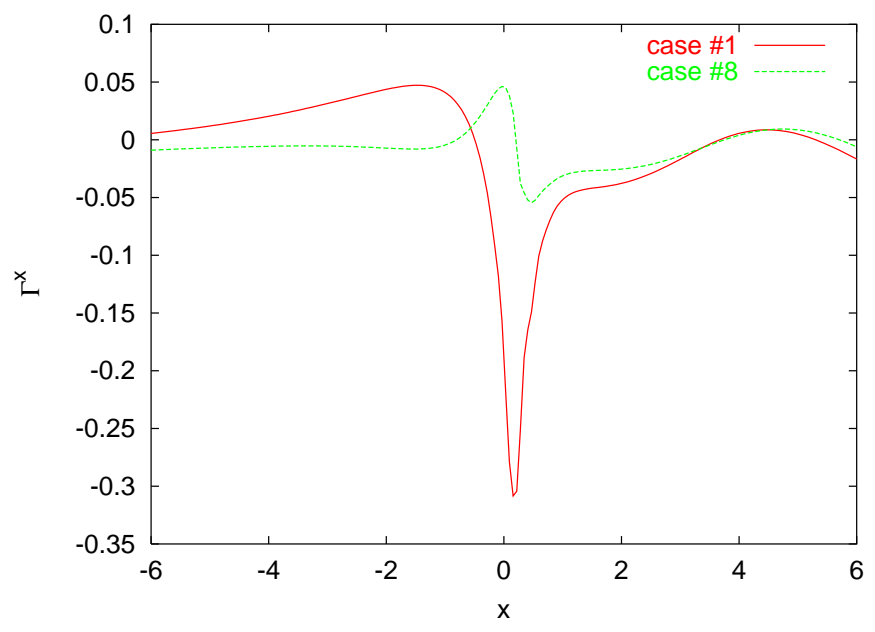

FIG. 11: $\tilde{\Gamma}^{x}$ for the gauge $\partial_{t} \alpha=-2 \alpha K+\beta^{j} \partial_{j} \alpha, \partial_{t} \beta^{i}=\frac{3}{4} B^{i}$, $\partial_{t} B^{i}=\partial_{t} \tilde{\Gamma}^{i}$ (Case \#1 with $\eta=0$ ) and for the gauge $\partial_{t} \alpha=$ $-2 \alpha K+\beta^{j} \partial_{j} \alpha, \partial_{t} \beta^{i}=\frac{3}{4} B^{i}+\beta^{j} \partial_{j} \beta^{i}, \partial_{t} B^{i}=\partial_{t} \tilde{\Gamma}^{i}+\beta^{j} \partial_{j}\left(B^{i}-\right.$ $\tilde{\Gamma}^{i}$ ) (Case $\# 8$ with $\eta=0$ ) at time $t=10 M$. In the "shiftingshift" case (Case \#8) $\tilde{\Gamma}^{x}$ is smaller and smoother around the puncture than in the "non-shifting-shift" case (Case \#1).

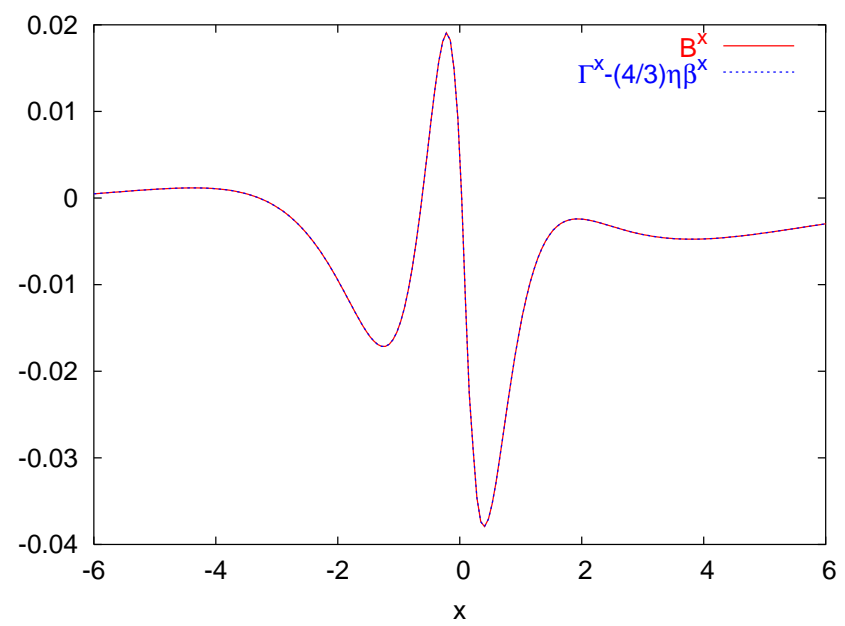

FIG. 12: Results for $B^{x}$ and $\tilde{\Gamma}^{x}-\frac{4}{3} \eta \beta^{x}$ with the gauge $\partial_{t} \alpha=$ $-2 \alpha K+\beta^{j} \partial_{j} \alpha, \partial_{t} \beta^{i}=\frac{3}{4} B^{i}+\beta^{j} \partial_{j} \beta^{i}, \partial_{t} B^{i}=\partial_{t} \tilde{\Gamma}^{i}+\beta^{j} \partial_{j}\left(B^{i}-\right.$ $\left.\tilde{\Gamma}^{i}\right)-\eta B^{i}$ at time $t=15 M$. The difference between the two curves is within $10^{-11}$.

since $B^{i}=\tilde{\Gamma}^{i}=\beta^{i}=0$ initially. This fact, also evident numerically (Fig. 12), suggests substituting for $B^{i}$ in the evolution equation for $\beta^{i}$, to obtain (in the case of Eqs. (21 22)

$$
\partial_{t} \beta^{i}=\frac{3}{4} \tilde{\Gamma}^{i}+\beta^{j} \partial_{j} \beta^{i}-\eta \beta^{i}
$$

Fig. 13] demonstrates that the resulting shift condition, Eq. (26), can be evolved numerically to yield the same stable, smooth simulation as with the analytically equivalent condition, Eqs. (21,22), used previously. Similar success should also be obtainable without the $\beta^{j} \partial_{j} \beta^{i}$

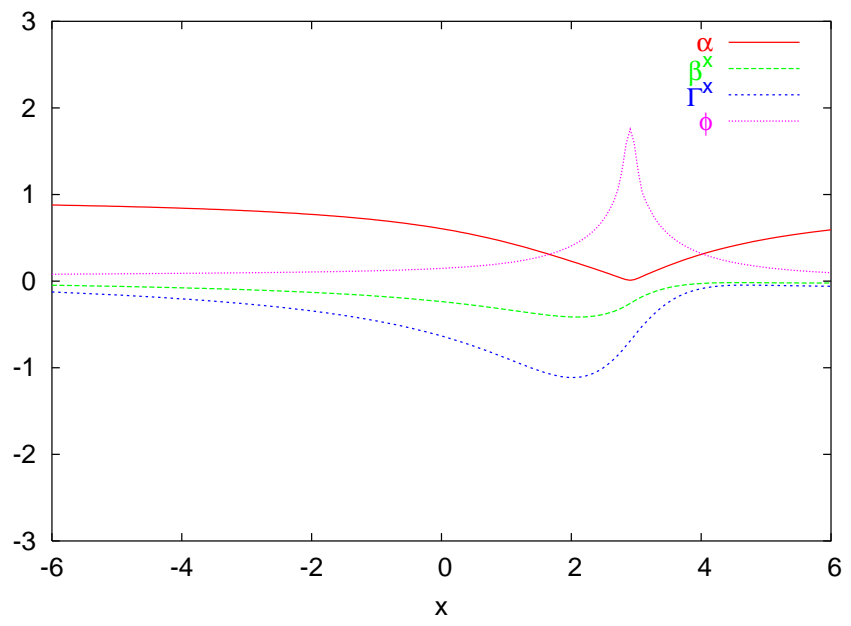

FIG. 13: Results for the gauge $\partial_{t} \alpha=-2 \alpha K+\beta^{j} \partial_{j} \alpha, \partial_{t} \beta^{i}=$ $\frac{3}{4} \tilde{\Gamma}^{i}+\beta^{j} \partial_{j} \beta^{i}-\eta \beta^{i}$ at time $t=30 M$. The results are nearly identical to those depicted in Fig. 10.

term. Dispensing with the $B^{i}$ evolution equation results in a slightly more efficient numerical implementation, and also guarantees removal of the zero-speed mode associated with $B^{i}$.

Finally it may be noted that when $\eta=0$ and $\tilde{\Gamma}^{i} \rightarrow 0$, Eq. (26) (and Eqs. (2122)) admits a "shock" solution of the form $\left(x-x_{0}\right) /\left(t_{0}-t\right)$. This represents the nonlinear tendency of this equation to advect large magnitudes of the shift faster than smaller magnitudes, which for negative values and a positive slope can lead to a vertical slope. However, as $\partial_{t} \tilde{\Gamma}^{i}$ depends on derivatives of $\beta^{i}, \tilde{\Gamma}^{i}$ is unlikely to vanish when those derivatives become large. Numerically $\beta^{i}$ has proven to be very well behaved so $\tilde{\Gamma}^{i}$ evidently acts as an effective "shock absorber", at the expense of not vanishing entirely.

\section{CONCLUSIONS}

We have investigated gauge conditions that are appropriate for the moving puncture approach to black hole simulations. Potential hazards from zero-speed or slowspeed modes have been identified, a methodology for analytically exploring various gauge choices has been presented, and a gauge free of slow-speed modes has been recommended. Several gauges were also studied numerically and two gauges distinguished themselves as particularly well-adapted to smooth black hole motion. In both cases we have suggested a simplification of the shift evolution equations, as well as explored the possibility of eliminating a traditional damping term to better realize a "Gamma-freezing" condition. We found that eliminating this damping term on the shift does indeed minimize $\tilde{\Gamma}^{i}$ and yield smooth evolutions, more so with the addition of our recommended "shifting-shift" terms, and warrants further experimentation. We intend to investigate this $\eta \rightarrow 0$ limit more thoroughly in future work. 


\section{Acknowledgments}

This work was supported in part by NASA grants ATP02-0043-0056 and O5-BEFS-05-0044. The simulations were carried out using Project Columbia at NASA Ames Research Center and at the NASA Center for Computational Sciences at Goddard Space Flight Center.
M.K and J.v.M. were supported by the Research Associateship Programs Office of the National Research Council and the NASA Postdoctoral Program at the Oakridge Associated Universities. We are very grateful to Carsten Gundlach for carefully reading and pointing out an error in an earlier draft.
[1] B. Bruegmann, W. Tichy, and N. Jansen, Phys. Rev. Lett. 92, 211101 (2004), gr-qc/0312112.

[2] M. Alcubierre et al., Phys. Rev. D72, 044004 (2005), gr-qc/0411149.

[3] J. G. Baker, J. Centrella, D.-I. Choi, M. Koppitz, and J. van Meter, Phys. Rev. Lett. 96, 111102 (2006), grqc/0511103.

[4] M. Campanelli, C. O. Lousto, P. Marronetti, and Y. Zlochower, Phys. Rev. Lett. 96, 111101 (2006), grqc/0511048.

[5] J. G. Baker, J. Centrella, D.-I. Choi, M. Koppitz, and J. van Meter, Phys. Rev. D73, 104002 (2006), grqc/0602026.

[6] T. Nakamura, K. Oohara, and Y. Kojima, Prog. Theor. Phys. Suppl. 90, 1 (1987).

[7] M. Shibata and T. Nakamura, Phys. Rev. D 52, 5428 (1995).

[8] T. W. Baumgarte and S. L. Shapiro, Phys. Rev. D 59, 024007 (1999), gr-qc/9810065.

[9] B. Imbiriba et al., Phys. Rev. D70, 124025 (2004), grqc/0403048.
[10] M. Alcubierre, B. Brügmann, P. Diener, M. Koppitz, D. Pollney, E. Seidel, and R. Takahashi, Phys. Rev. D 67, 084023 (2003), gr-qc/0206072.

[11] C. Bona, J. Masso, E. Seidel, and J. Stela, Phys. Rev. Lett. 75, 600 (1995), gr-qc/9412071.

[12] S. Brandt and B. Brügmann, Phys. Rev. Lett. 78, 3606 (1997), gr-qc/9703066.

[13] J. D. Brown and L. L. Lowe, J. Comput. Phys. 209, 582 (2005), gr-qc/0411112.

[14] P. A. M. Dirac, Phys. Rev. 114, 924 (1959).

[15] L. Smarr and J. W. York, Phys. Rev. D17, 1945 (1978).

[16] M. Alcubierre, G. Allen, B. Brügmann, E. Seidel, and W.-M. Suen, Phys. Rev. D 62, 124011 (2000), grqc/9908079.

[17] M. Campanelli, C. O. Lousto, and Y. Zlochower, Phys. Rev. D 73, 061501 (2006), gr-qc/0601091.

[18] C. Gundlach and J. M. Martin-Garcia (2006), grqc/0604035.

[19] H. Beyer and O. Sarbach, Phys. Rev. D70, 104004 (2004), gr-qc/0406003. 